\title{
Mortalidad por infarto agudo al miocardio en Chile: 1990-2001
}

\author{
Jorge Szot Meza
}

\author{
Mortality caused by acute \\ myocardial infarction in Chile in the \\ period 1990-2001
}

Background: Acute myocardial infarction is the first cause of death among adults in Chile. It caused 5,650 deaths during 2001. Aim: To analyze the evolution and mortality of Acute Myocardial Infarction in Chile between 1990 and 2001. Material and methods: Analysis of data on mortality caused by Acute Myocardial Infarction in Chile, obtained from the databases of the Ministry of Health. The adjusted mortality rates and disability adjusted life years (DALY) were calculated for the period. Results: In the study period, adjusted mortality decreased from 86 to 67.6 deaths/100,000 inhabitants. The DALYs ratio between men and women increased from 2.7 to 2.9. The mean death age for men and women was 69.9 and 76.5 years respectively ( $p<0.001$ ). Forty five percent of women and $51 \%$ of men died at home, $78 \%$ of women and $66 \%$ of men received medical attention. Conclusions: $\mathrm{Al}-$ though there was a reduction in mortality caused by acute myocardial infarction in the study period, there are still gender differences in the age of death, and access to medical care (Rev Méd Chile 2004; 132: 1227-33).

(Key Words: Cause of deaths; Myocardial infarction; Sick leave)

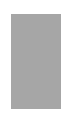

Recibido el 15 de abril, 2004. Aceptado en versión corregida el 5 de agosto, 2004.

Departamento de Epidemiología, División de Rectoría y Regulación Sanitaria, Ministerio de Salud de Chile.

L as enfermedades cardiovasculares y las neo_plasias dan cuenta de la mitad de las defunciones ocurridas en el país desde mediados de la década de los años 80 en adelante ${ }^{1,2}$. Lo anterior, tiene que ver con que la población del país envejece con celeridad $\mathrm{y}$, por tanto, al vivir las personas un mayor número de años, se encuentran expuestas por más tiempo a los factores de riesgo conocidos para esas patologías 3 . Durante el año 2001, ocurrieron en el país 22.373 defunciones por causa cardiovascular (Código: CIE-X, I 00 - I 99) ${ }^{4}$,

Correspondencia a: Dr. Jorge Szot M. Mac Iver 541, 4ํㅜ piso, Santiago. Teléfono: 6300481. Fax: 6300505.

E mail: jszot@minsal.gov.cl constituyendo el 27\% del total de muertes. Dentro de este grupo, el infarto agudo al miocardio (IAM) (CIE-X, I 21) y la enfermedad isquémica crónica del corazón (CIE-X, I 25) produjeron, en conjunto, 7.403 defunciones (33\%). El accidente vascular encefálico (CIE-X, I 64) y la hemorragia intraencefálica sumados (CIE-X, I 61) ocasionaron 4.478 defunciones (20\%). La hipertensión arterial esencial primaria (CIE-X, I 11) 1.001 defunciones (4,5\%). El resto de las causas cardiovasculares contribuyen en una proporción inferior a la mortalidad por estas patologías.

Dado que la causa aislada que produjo la mayor cantidad de defunciones fue el IAM, se presenta este trabajo, que da cuenta de las 
características de la mortalidad por esta enfermedad en el país entre 1990 y el año 2001.

\section{MATERIAL Y MÉTODO}

Estudio epidemiológico descriptivo. Los datos sobre mortalidad fueron obtenidos de las bases de defunciones del Departamento de Estadísticas e Información en Salud del Ministerio de Salud. No se tuvo acceso a los certificados médicos de defunción de estos pacientes. Los datos obtenidos, para los años 1990-2001 fueron: edad de muerte, sexo, diagnóstico de la causa primaria de muerte, que en este caso fue IAM (Códigos: CIE-IX, 410, CIE-X, I 21) en personas mayores de 15 años y localización urbano-rural de la defunción. Con éstos, se calcularon tasas específicas de mortalidad (TM) para IAM. Estas se ajustaron usando como población de referencia la población chilena, mayor de 15 años, según el Censo del año $2002^{5}$. También se calculó la cantidad de Años de Vida Potencial Perdidos (AVPP) ${ }^{6}$ en cada año, expresado en tasa por 1.000 habitantes. Para los años 1998 a 2001, fue posible obtener además, datos sobre nivel de instrucción del paciente fallecido, actividad desarrollada, atención médica al momento de ocurrir la defunción y lugar de fallecimiento.

Estadística. Los datos se analizaron, usando la prueba de $t$ de Student para comparación de medias independientes, en el caso de las variables numéricas y el test de chi cuadrado, para la comparación de variables nominales. La significación estadística se fijó con un valor $\mathrm{p} \leq 0,05$. Con los datos de las defunciones por IAM del año 2001, se construyó un mapa de TM por esta causa en las regiones de Chile, ajustando la mortalidad de cada región, usando como población de referencia la población del Censo 2002.

El cálculo de los AVPP se efectuó como sigue:

\begin{tabular}{|l|}
\hline L \\
AVPP: $\sum(\mathrm{L}-\mathrm{Xi})$ \\
$\mathrm{x}=0$ \\
\hline
\end{tabular}

donde:

$\mathrm{L}=$ Edad límite potencial de la vida (80,0 años para hombres y 82,5 años para mujeres).

$\mathrm{X}=$ Edad de defunción del sujeto.

Los software utilizados fueron el SPSS versión 10.0 para el análisis estadístico y el Arc View versión 3.2 para la elaboración del mapa geográfico.

\section{RESUlTADOs}

Para el período comprendido entre los años 1990 y 2001, en la Figura 1 se observa la baja en la mortalidad por IAM en ambos sexos. En las Figuras 2 y 3 se aprecia que la tasa de mortalidad no

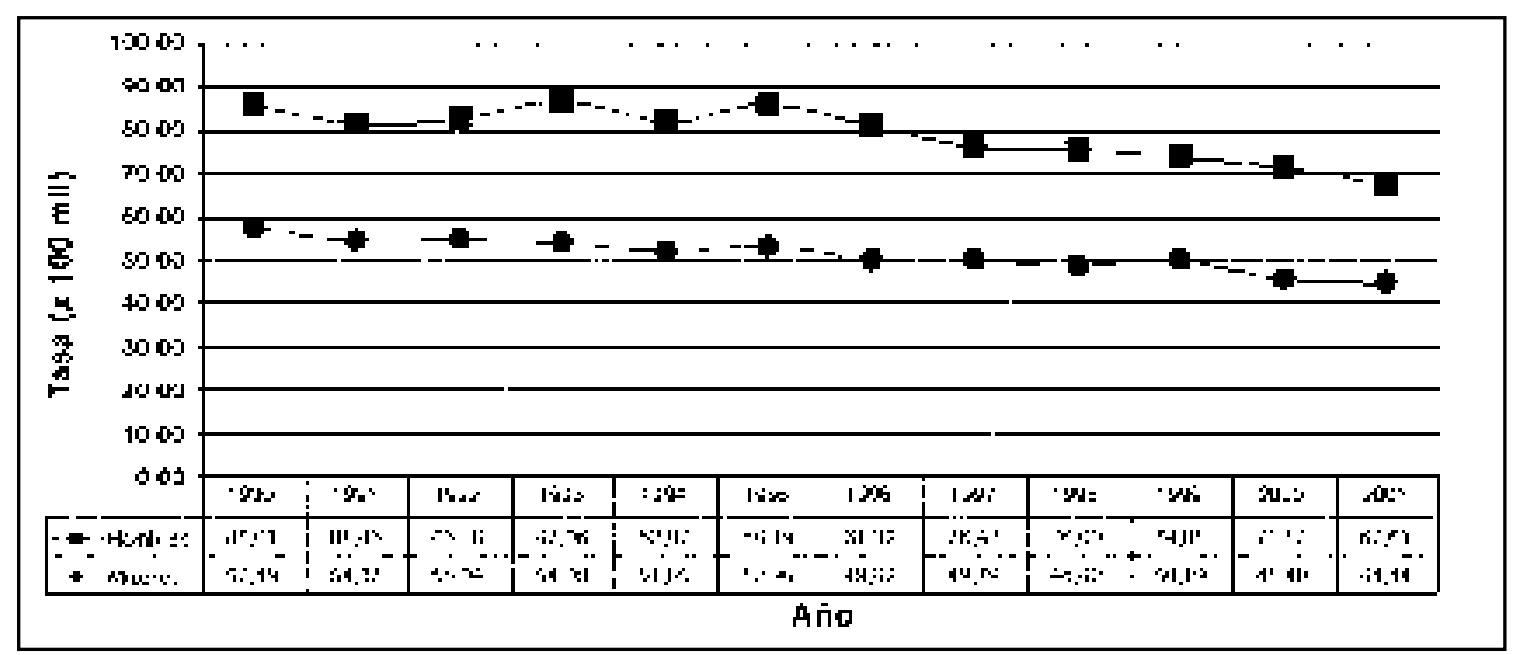

FIGURA 1. Evolución de la tasa de mortalidad ajustada por infarto agudo al miocardio, Chile, 1990-2001. 


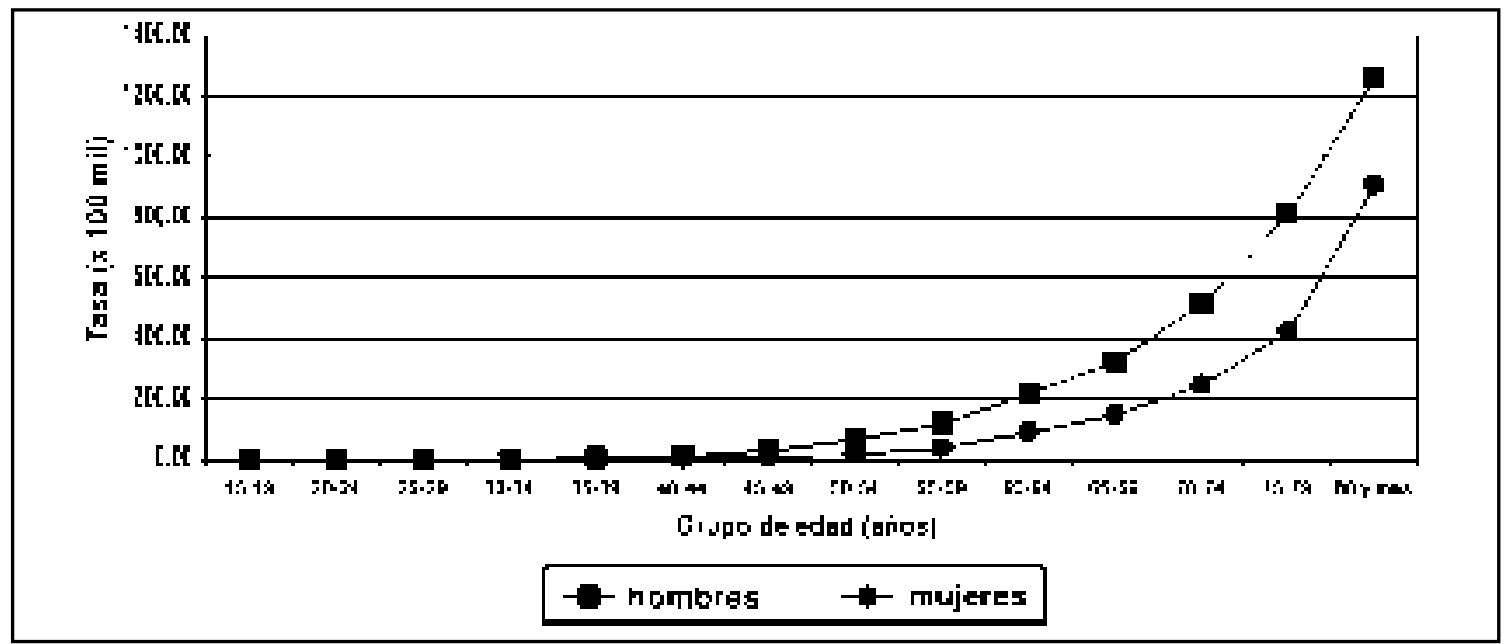

FIgURA 2. Distribución de la mortalidad por infarto agudo al miocardio según grupos quinquenales de edad, Chile, 1990.

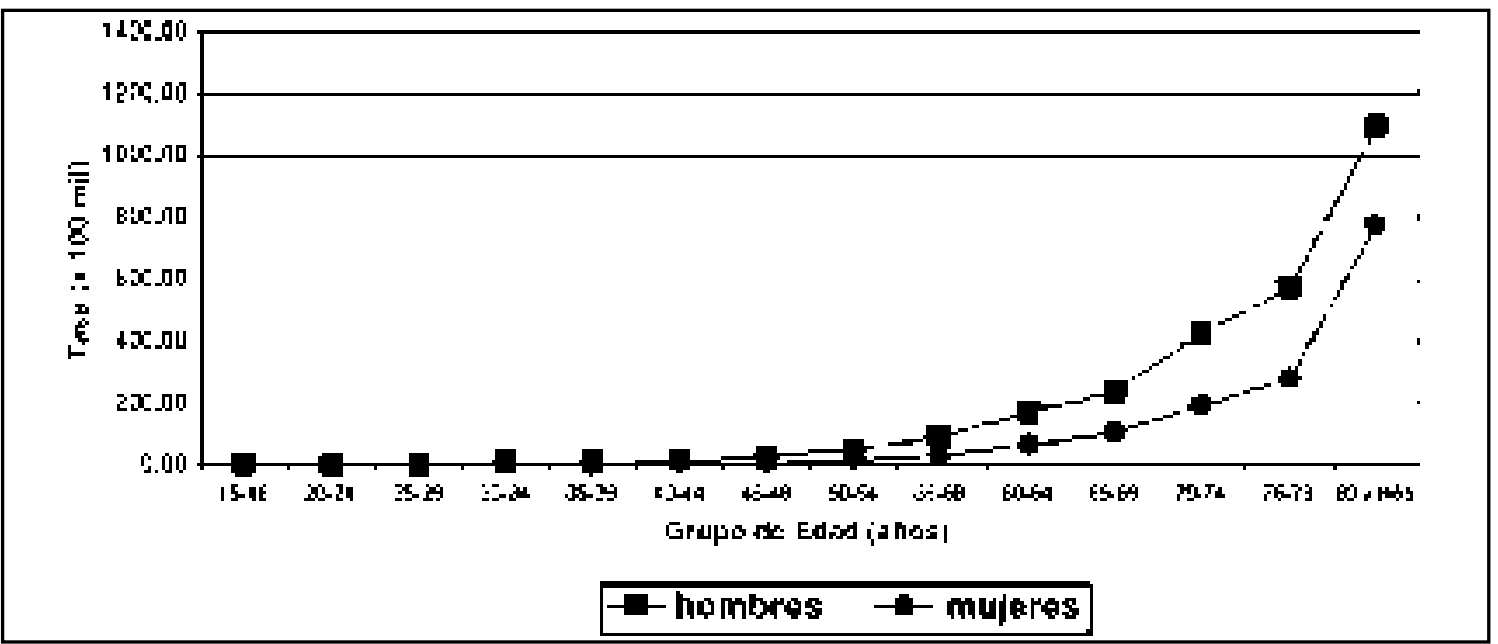

FiguRA 3. Distribución de la mortalidad por infarto agudo al miocardio según grupos quinquenales de edad, Chile, 2001.

ajustada por IAM fue mayor en hombres que en mujeres, especialmente a partir de los 55 años. En la Figura 4, la distribución por sexo de las defunciones se muestra el predominio que éstas tienen en hombres. Los AVPP por IAM en hombres fueron mayores que los AVPP en mujeres: Figura 5. En la Tabla 1, la comparación de variables seleccionadas, destaca que las defunciones por IAM se concentraron en hombres, con escolaridad básica, y a una edad menor que en mujeres. Los pacientes fallecieron en una proporción similar en su casa y en el hospital, excepto las mujeres, que fallecieron más en el hogar. En la Figura 6, el mapa de la TM por IAM en las regiones de Chile, se muestra que la tasa más alta de mortalidad por IAM estuvo en la región de Aysén.

\section{Discusión}

En cifras absolutas, la cantidad total de personas fallecidas por IAM se ha mantenido relativamente 


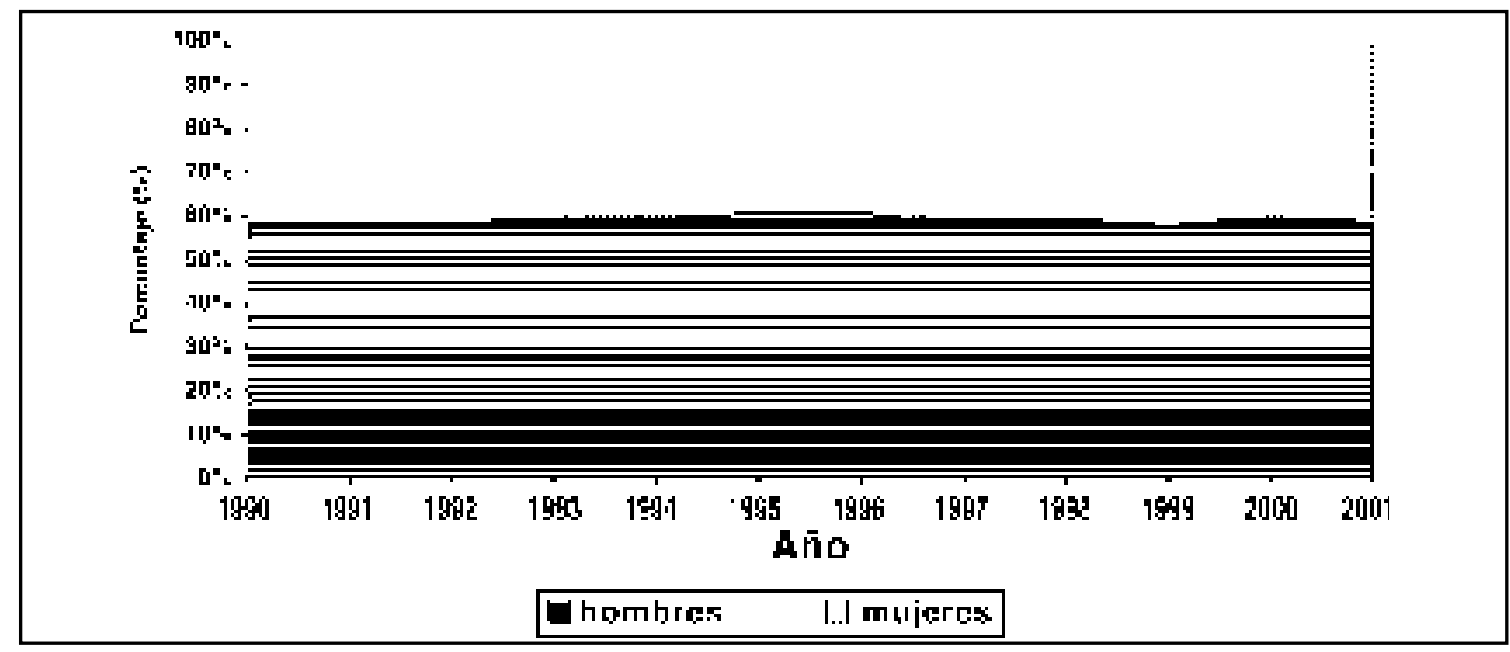

FiguRA 4. Distribución de la mortalidad por infarto agudo al miocardio según sexo, Chile, 1990-2001.

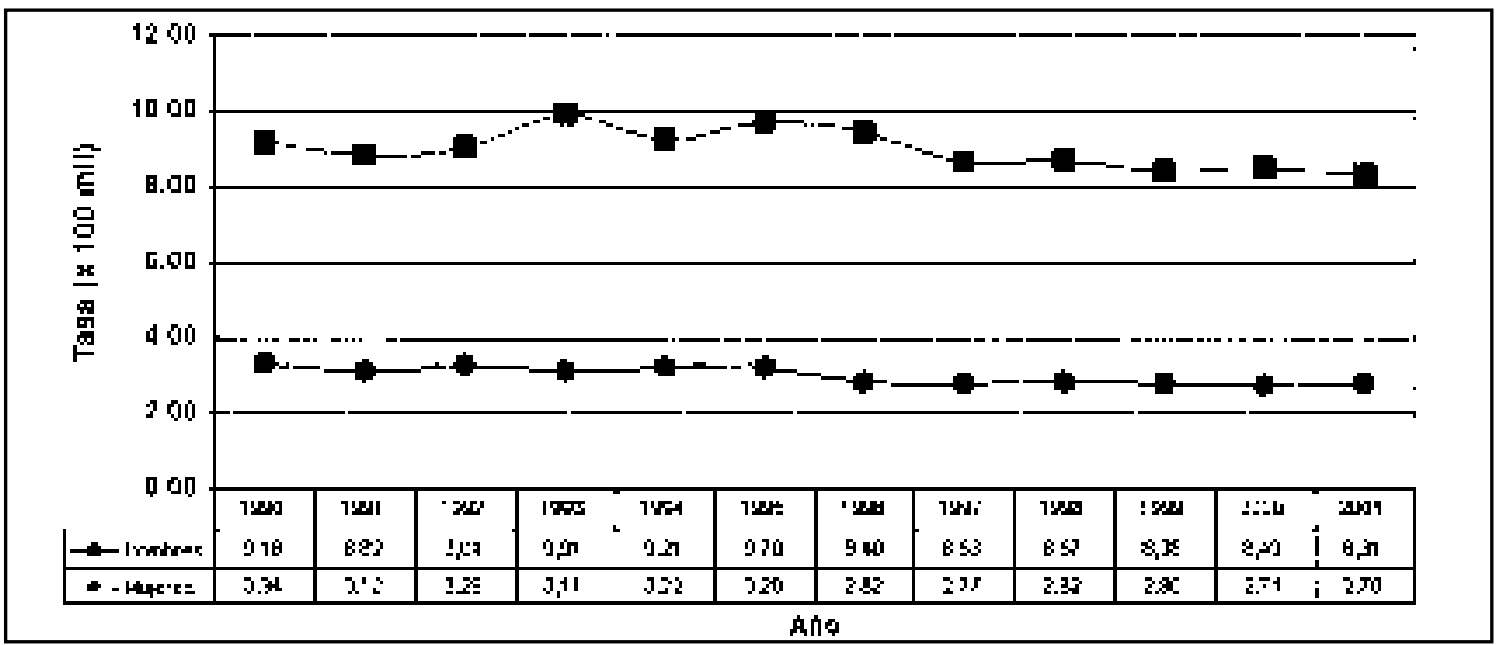

FiguRA 5. Evolución de la tasa de AVPP por infarto agudo al miocardio, Chile, 1990-2001.

constante en Chile, entre 1990 y 2001, en torno a los 5.500 eventos al año. Sin embargo, luego de ajustar las tasas de mortalidad (TM), se observa una reducción en el riesgo de fallecer por esta causa de $21 \%$ en hombres, de una TM de 86,01 por 100 mil en 1990 a una TM de 67,61 por 100 mil en 2001 y de $22 \%$ en mujeres, de una TM de 57,49 por 100 mil en 1990 a una TM de 44,44 por 100 mil en 2001 (Figura 1). Esta baja en la TM puede asociarse a múltiples causas, algunas de las cuales tienen que ver con el mejoramiento en el diagnóstico y con la introducción de avances terapéuticos, especialmente en lo que se refiere al uso de nuevos fármacos, desde fines de los años noventa 7,8 .

Al comparar la situación de mortalidad por IAM de Chile, con la de países con una situación demográfico-epidemiológica similar, como Argentina y Uruguay, se observa que durante 2001 la tasa de mortalidad por IAM de Chile, de 51 por 100 mil para ambos sexos, coincide con la tasa de mortalidad por IAM en Argentina, de 54 por 100 
Tabla 1. Comparación de variables seleccionadas en pacientes fallecidos por infarto agudo al miocardio, Chile, 1998-2001.

\begin{tabular}{|c|c|c|c|c|c|c|c|}
\hline $\begin{array}{l}\text { Variable (\%) } \\
1998\end{array}$ & $\begin{array}{l}\text { hombres } \\
(n=3.379)\end{array}$ & $\begin{array}{l}\text { mujeres } \\
(\mathrm{n}=2.327)\end{array}$ & valor-p & 2000 & $\begin{array}{l}\text { hombres } \\
(n=3.383)\end{array}$ & $\begin{array}{l}\text { mujeres } \\
(n=2.305)\end{array}$ & valor-p \\
\hline $\begin{array}{l}\text { Edad promedio (años)* } \\
\text { Nivel instrucción }\end{array}$ & $70,0 \pm 12,8$ & $76,5 \pm 12,1$ & 0,007 & $\begin{array}{l}\text { Edad promedio } \\
\text { Nivel instrucción }\end{array}$ & $69,9 \pm 12,7$ & $76,3 \pm 11,9$ & 0,000 \\
\hline Básico & 54,0 & 60,9 & 0,000 & Básico & 56,9 & 61,6 & 0,000 \\
\hline Secundario & 29,8 & 20,8 & & Secundario & 26,7 & 19,8 & \\
\hline Superior & 5,5 & 2,4 & & Superior & 6,4 & 2,0 & \\
\hline Otro & 10,7 & 15,9 & & Otro & 10,0 & 16,6 & \\
\hline Total & 100,0 & 100,0 & & Total & 100,0 & 100,0 & \\
\hline Actividad & & & & Actividad & & & \\
\hline Inactivo & 71,4 & 98,0 & 0,000 & Inactivo & 71,4 & 98,0 & 0,000 \\
\hline Activo & 26,8 & 1,8 & & Activo & 26,5 & 2,0 & \\
\hline Otro & 1,8 & 0,2 & & Otro & 2,1 & 0,0 & \\
\hline Total & 100,0 & 100,0 & & Total & 100,0 & 100,0 & \\
\hline Atención médica & & & & Atención médica & & & \\
\hline Sí & 66,2 & 78,3 & 0,000 & Sí & 65,0 & 76,4 & 0,000 \\
\hline No & 23,4 & 16,7 & & No & 22,6 & 17,1 & \\
\hline Ignorada & 10,4 & 5,0 & & Ignorada & 12,4 & 6,5 & \\
\hline Total & 100,0 & 100,0 & & Total & 100,0 & 100,0 & \\
\hline Lugar defunción & & & & Lugar de defunción & & & \\
\hline Hospital & 43,9 & 43,6 & 0,000 & Hospital & 43,0 & 43,6 & 0,000 \\
\hline Casa & 44,3 & 51,5 & & Casa & 44,8 & 49,9 & \\
\hline Otro & 11,8 & 4,9 & & Otro & 12,2 & 6,5 & \\
\hline Total & 100,0 & 100,0 & & Total & 100,0 & 100,0 & \\
\hline 1999 & $\begin{array}{l}\text { hombres } \\
(n=3.390)\end{array}$ & $\begin{array}{l}\text { mujeres } \\
(n=2.465)\end{array}$ & valor-p & 2001 & $\begin{array}{l}\text { hombres } \\
(n=3.317)\end{array}$ & $\begin{array}{c}\text { mujeres } \\
(n=2.333)\end{array}$ & valor-p \\
\hline Edad promedio (años)* & $70,4 \pm 12,7$ & $76,8 \pm 11,7$ & 0,000 & Edad promedio & $69,6 \pm 12,8$ & $76,4 \pm 12,2$ & 0,033 \\
\hline Nivel instrucción & & & & Nivel instrucción & & & \\
\hline Básico & 55,5 & 61,2 & 0,000 & Básico & 56,2 & 64,8 & 0,000 \\
\hline Secundario & 27,6 & 19,7 & & Secundario & 26,3 & 18,4 & \\
\hline Superior & 6,0 & 2,7 & & Superior & 7,2 & 2,2 & \\
\hline Otro & 10,9 & 16,4 & & Otro & 10,3 & 14,6 & \\
\hline Total & 100,0 & 100,0 & & Total & 100,0 & 100,0 & \\
\hline Actividad & & & & Actividad & & & \\
\hline Inactivo & 72,4 & 97,9 & 0,000 & Inactivo & 72,4 & 97,7 & 0,000 \\
\hline Activo & 26,1 & 2,1 & & Activo & 25,6 & 2,1 & \\
\hline Otro & 1,5 & 0,0 & & Otro & 2,0 & 0,2 & \\
\hline Total & 100,0 & 100,0 & & Total & 100,0 & 100,0 & \\
\hline Atención médica & & & & Atención médica & & & \\
\hline $\mathrm{Sí}^{\prime}$ & 66,4 & 78,0 & 0,000 & Sí & 66,9 & 78,7 & 0,000 \\
\hline No & 21,9 & 16,9 & & No & 21,3 & 15,7 & \\
\hline Ignorada & 11,7 & 5,1 & & Ignorada & 11,8 & 5,6 & \\
\hline Total & 100,0 & 100,0 & & Total & 88,2 & 94,4 & \\
\hline Lugar de defunción & & & & Lugar de defunción & & & \\
\hline Hospital & 42,9 & 41,2 & 0,000 & Hospital & 42,1 & 42,5 & 0,000 \\
\hline Casa & 45,5 & 52,9 & & Casa & 45,0 & 50,5 & \\
\hline Otro & 11,6 & 5,9 & & Otro & 12,9 & 7,0 & \\
\hline Total & 100,0 & 100,0 & & Total & 100,0 & 100,0 & \\
\hline
\end{tabular}

*y desviación estándar 


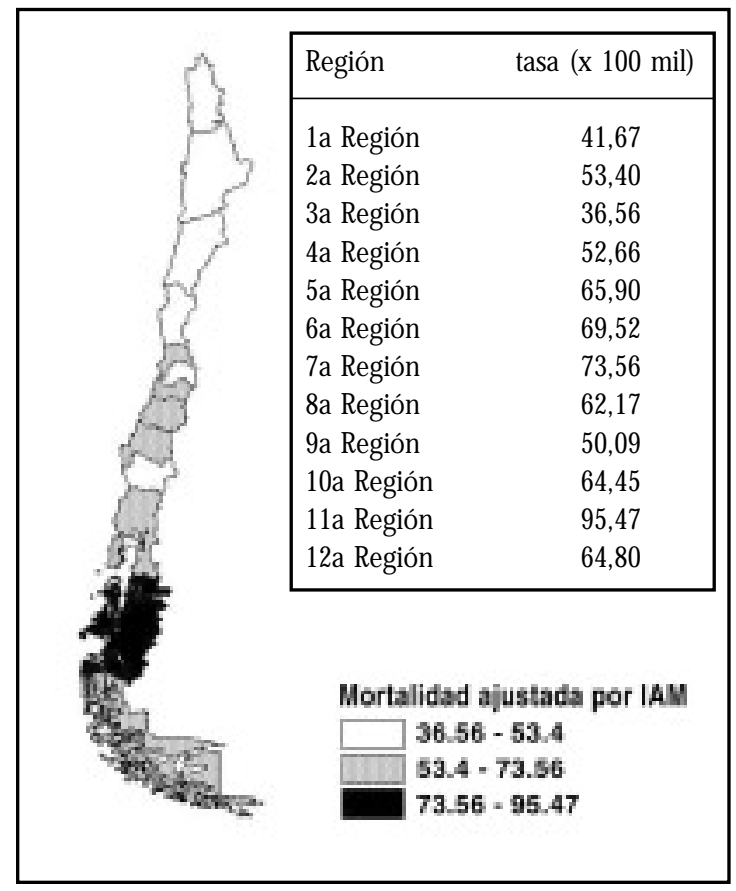

Figura 6. Tasa de mortalidad ajustada por infarto agudo al miocardio, según región. Chile, 2001.

mil para ambos sexos ${ }^{10}$ pero es la mitad que la tasa de mortalidad por IAM de Uruguay, de 100 por 100 mil para ambos sexos ${ }^{9}$. En estos países también la mortalidad por IAM muestra una tendencia decreciente ${ }^{9}$.

Si bien, el análisis de la mortalidad por edad y sexo muestra que para todos los grupos de edad la letalidad por IAM en la población general es más alta en hombres que en mujeres (Figuras 2, 3 y 4), a nivel hospitalario ocurre la situación opuesta. Datos reportados por el Registro de Infarto Agudo al Miocardio en Hospitales Chilenos (Grupo GEMI) ${ }^{10}$ muestran que la letalidad de pacientes es mayor en mujeres que en hombres. En este registro se demostró, que dentro de los factores predictores independientes de mortalidad por IAM al ingreso de los pacientes, está el sexo femenino junto con la edad avanzada, la presencia de diabetes mellitus, la localización anterior del infarto y la presencia de insuficiencia cardíaca ${ }^{11}$.

Ahora bien, la mortalidad precoz por IAM, expresada a través de los años de vida potencial perdidos (AVPP), muestra que el promedio de la tasa de AVPP en hombres triplica el promedio de la tasa de AVPP en mujeres: 8,97 AVPP por 1.000 versus 3,00 AVPP por 1.000, respectivamente. No obstante ambos indicadores disminuyeron en el período, la mayor baja se produjo en el sexo femenino con $17 \%$, versus sólo $9 \%$ en el sexo masculino (Figura 5). Esto se asocia al hecho que la letalidad en hombres se produce a edades menores que las mujeres, con lo que la magnitud de los años de vida perdidos en forma precoz en ellos es mayor.

Comparando algunas variables seleccionadas de la mortalidad por IAM en ambos sexos (para los que sólo existe registro entre 1998 y 2001), se encuentran hallazgos de interés. Es así como la edad de defunción en el caso de los hombres es consistente y significativamente menor que la edad de defunción en el caso de las mujeres: 69,9 años en promedio en sexo masculino versus 76,5 años en promedio en sexo femenino $(p=0,000)$. Respecto del nivel educacional de los pacientes fallecidos, más de la mitad de las defunciones ocurrieron en personas que sólo tenían escolaridad básica: 55,6\% en hombres y $62,1 \%$ en mujeres, situación que también ha sido comunicada en el extranjero ${ }^{12}$. Esto, se relaciona con una alta prevalencia de factores de riesgo considerados como de peor pronóstico para la evolución del IAM como son: la dislipidemia y la diebetes mellitus ${ }^{12,13}$, especialmente asociadas a la obesidad, que en general, se encuentra en mayor proporción en personas con menor nivel de educación ${ }^{12,14}$.

Se encontró que la mayor cantidad de pacientes pertenecía a la categoría ocupacional «nactivo». $71,9 \%$ de los hombres y $97,9 \%$ de las mujeres lo que tiene que ver con la edad en la que ocurren estas defunciones.

Un hecho que llama la atención de las comparaciones efectuadas, es que la atención médica (AM) al momento de fallecer estuvo presente en una proporción significativamente menor en hombres que en mujeres: $66,1 \%$ de los hombres fallecidos por IAM versus $77,8 \%$ de las mujeres fallecidas recibieron AM $(p=0,000)$ aunque la proporción de hombres y de mujeres que fallece en hospitales es prácticamente la misma: $42,9 \%$ y $42,7 \%$. Lo anterior puede relacionarse con el hecho que $12,2 \%$ de los hombres fallece en un lugar distinto al hospital o su domicilio (ejemplo: vía pública) versus sólo $6 \%$ de las mujeres y 
también con que las mujeres que fallecen por IAM tienen mayor edad que los hombres, pudiendo encontrarse bajo supervisión médica más permanente. En todo caso, se trata de una situación que no ha sido comunicada previamente en nuestro medio.

Respecto de la mortalidad por IAM en las regiones de Chile, durante el año 2001 se encontró las tasas más bajas en las regiones de Tarapacá y Metropolitana y la tasa más alta en la región de Aysén. Aunque lo anterior puede relacionarse con las condiciones de acceso a una atención médica oportuna, sería necesario observar cómo ha sido la tendencia de la distribución geográfica de las defunciones por IAM en el país en un período mayor de tiempo, de forma de obtener una

\section{REFERENCIAS}

1. Albala C, Vio F, Robledo A, Icaza G. La Transición Epidemiológica en Chile. Rev Méd Chile 1993; 121: 1446-5.

2. Szot J. Transición Demográfico-Epidemiológica en Chile. Rev Esp Salud Pública 2003; 77: 605-13.

3. Berríos X, Jadue L, Centeno J. Prevalencia de Factores de Riesgo de Enfermedades Crónicas. Estudio en la Población General de la Región Metropolitana, 1986-1987. Rev Méd Chile 1990; 118: 597-604.

4. Base de Datos de Mortalidad 2001, Departamento de Estadísticas e Información en Salud (DEIS), División de Rectoría y Regulación Sanitaria, Ministerio de Salud, Chile.

5. Sitio URL: http://deis.minsal.cl/deis/pobla/ cuadro1_2_00000.htm

6. MurRay CJL. Cuantificación de la Carga de Enfermedad: la base técnica del cálculo de los años de vida ajustados en función de la discapacidad. Bol Oficina Sanit Panam 1995; 118: 221-41.

7. Lanas F, Prieto J, Chávez E, Corbalán R, Cumsile F. Uso de bloqueadores beta adrenérgicos en el infarto del miocardio. Rev Chil Cardiol 1998; 17: 205-12. conclusión más definitiva. También, es posible que la distribución de factores de riesgo cardiovascular sea mayor en algunas regiones de Chile que en otras, lo que podría asociarse con una mayor mortalidad por IAM. En este sentido, será de gran utilidad, la información que arroje la primera Encuesta Nacional de Salud del Ministerio de Salud.

El presente trabajo pretende contribuir al mejor conocimiento de las características que ha tenido la mortalidad de la principal causa aislada de defunción en el país durante la última década. De este modo, se quiere mostrar una tendencia que permita proyectar, de no producirse cambios significativos en la magnitud de factores de riesgo para esta enfermedad, el comportamiento futuro de ella.
8. Prieto J, Corbalán R, Nazzal C, Chávez E, lanas F, Bartolucci J et al. Cambios en los patrones de prescripción de medicamentos en el infarto agudo del miocardio: Comparación de dos períodos. Rev Méd Chile 2001: 129; 481-8.

9. CuRTo S. Mortalidad por enfermedades cardiovasculares en el Uruguay. Bol Acad Nac Med B Aires 1998; 76: 537-49.

10. sitio URL: http://www.msal.gov.ar/htm/site/ estadisticas.asp

11. Corbalán R, Nazzal C, Prieto J, Chávez E, Lanas F, Lamich R, Bartolucci J et al. Reducción de la mortalidad por infarto del miocardio en hospitales chilenos. Rev Méd Chile 2002; 130: 368-78.

12. Coelho R, Ramos E, Prata J, Maciel MJ, Barros H. Acute myocardial infarction: psychosocial and cardiovascular risk factors in men. J Cardiovasc Risk 1999; 3: 157-62.

13. Tavani A, Bertuzzi M, Galuus S, Negri E, La Vecchia C. Diabetes mellitus as a contributor to the risk of acute myocardial infarction. J Clin Epidemiol 2002; 55: 1082-7.

14. Rivera JA, Barquera S, Campirano F, Campos I, Safdie M, Tovar V. Epidemiological and nutritional transition in Mexico: rapid increase of non-communicable chronic diseases and obesity. Public Health Nutr 2002; 5: 113-22. 